\title{
A CODE FOR CALCULATING STATIC MODEL STELLAR ATMOSPHERES
}

\author{
M. I. NOuH* \\ National Research Institute of Astronomy and Geophysics, 11722, Helwan, Cairo, Egypt \\ E-mail: nouh@nriag.sci.eg \\ (Received March 27, 2009; Accepted June 1, 2009)
}

\begin{abstract}
In this paper we present an independent FORTRAN code for calculating LTE-plane-parallel model atmospheres. The transfer equation has been solved using Avrett and Loeser method. It is shown that, using an approximate non-gray temperature distribution together with the iteration factors method (Simonneau and Crivellari) for correcting the temperature distribution reduce the number of iteration required to achieve the condition of radiative equilibrium. Preliminary results for pure helium model atmospheres are presented.
\end{abstract}

Key words : Stars: Stellar atmosphere, Fundamental Parameters; Methods: Numerical

\section{INTRODUCTION}

Stellar atmospheres can be considered as laboratories of plasma physics, in which the atomic parameters can be tested. Also the mathematical methods developed in stellar atmospheres can be widely used in many other problems in astronomy (Mihalas, 1978).

In astrophysics, one of the importance of modelling stellar atmosphere is that it provides an adequate surface boundary condition for the computation of evolutionary stellar models. Besides, the atmosphere models are necessary to determine the emergent flux from the star, which is required to interpret the luminosities and colors of observed stellar spectra.

Two alternative approaches are possible for a numerical solution of the problem of modelling stellar atmosphere; a global approach (e.g. complete liberalization) and iterative methods.

In the 1970s two basic categories of model atmospheres were developed: the LTE line-blanketed models of Kurucz (1979), and the non-LTE models pioneered by Auer \& Mihalas (1972). Discussion of more developments could be found in Kudritzki (1988) and Hubeny (1997).

Although ATLAS code (Kurucz, 1979) shears with our code in static plane-parallel, LTE and radiative equilibrium conditions, our code is different from ATLAS in two situations, the temperature correction procedure and the integration scheme of the flux and mean intensity integrals.

For temperature correction algorithm we implemented Simonneau and Crivellari (1988) method, while the analytical expressions by Klinglesmith (1967) are adopted for the solution of the flux and mean intensity

\footnotetext{
* Present Address: Faculty of Science, Northern Borders Univer-
} sity, Arar, Saudi Arabia integrals.

The aim of the present paper is to carry out various calculations of LTE-plane parallel model atmospheres using an independent FORTRAN code. Preliminary results for pure helium model atmospheres are presented and compared with previous work.

The organization of the paper is as follows: Section 2 deals with the formulation of the basic equations, Section 3 devoted to the computational procedure and numerical methods used to compute models, Section 4 presents the whole procedure in a stepwise fashion, finally the results and conclusion reached are given in Section 5 .

\section{BASIC EQUATIONS}

In the present paper we assume that the atmosphere is plane-parallel (pp) and in local thermodynamic equilibrium (LTE). It has two input parameters which characterize the atmosphere, the effective temperature, $T_{\text {eff }}$ and the logarithm of the surface gravity $\log g$.

To construct model atmospheres (concerning the above assumptions), a set of equations must be solved, these equations are:

- The hydrostatic equilibrium equation which determines the gas pressure $p_{g}$.

- The electron-temperature relation, determining the electron pressure, $p_{e}$.

- The radiative transfer equation which are solved for the mean intensity integral, $J_{i}$, flux integral, $F_{i}, i=1, \ldots . N F$ on a pre-chosen frequency grid comprising $N F$ points.

- The radiative equilibrium for which the temperature follows. 
This set of equations has to be solved at each point $d$ of a grid comprising $N D$ depth points. In the following, the basic equations needed for constructing model atmosphere will be formulated .

\section{(a) Hydrostatic Equilibrium} form

We use the hydrostatic equilibrium equation in the

$$
\frac{d \log P_{g}}{d \tau}=\frac{1}{2.302585 P_{g}}\left(\frac{g}{\left(k_{\nu}+\sigma_{\nu}\right)_{s}}-\frac{d P_{r}}{d \tau}\right),
$$

where $P_{g}$ is the gas pressure, $g$ the surface gravity, $k_{\nu}$ and $\sigma_{\nu}$ are the mass absorption coefficient and the scattering coefficient at frequency $\nu, P_{r}$ the radiation pressure. The subscript $s$ denotes the opacity determined at a standard frequency (s) (4000 $\AA$ for the present calculations) and $d p_{r} / d \tau$ is given by

$$
\frac{d p_{r}}{d \tau}=\frac{\pi}{c} \int_{0}^{\infty} \frac{k_{\nu}+\sigma_{\nu}}{(k+\sigma)_{s}} F_{\nu} d \nu
$$

\section{(b) Radiative Transfer}

The transfer equation in plane-parallel geometry could be written as

$$
\mu \frac{d I_{\nu}}{d \tau_{\nu}}=I_{\nu}-S_{\nu}
$$

where $\mu=\cos \theta, d \tau_{\nu}=-\left(k_{\nu}+\sigma_{\nu}\right) \rho d z, \theta, I_{\nu}$ and $S_{\nu}$ are the angle of the emergent radiation relative to the normal to the star surface, specific intensity and source function at frequency $\nu$ respectively. The formal solution of Equation (3) gives the full intensity at the position $\tau_{\nu}$ as

$$
\begin{aligned}
I_{\nu}\left(\tau_{\nu}\right) & =\int_{\tau_{\nu}}^{\infty} S_{\nu}\left(t_{\nu}\right) e^{-\left(t_{\nu}-\tau_{\nu}\right) / \mu} d t_{\nu} / \mu \\
& -\int_{0}^{\tau_{\nu}} S_{\nu}\left(t_{\nu}\right) e^{-\left(t_{\nu}-\tau_{\nu}\right) / \mu} d t_{\nu} / \mu .
\end{aligned}
$$

After some manipulations the monochromatic flux at each optical depth has the form

$$
\begin{aligned}
F_{\nu}\left(\tau_{\nu}\right) & =2 \pi \int_{\tau_{\nu}}^{\infty} S_{\nu} E_{2}\left(t_{\nu}-\tau_{\nu}\right) d t_{\nu} \\
& -2 \pi \int_{0}^{\tau_{\nu}} S_{\nu} E_{2}\left(\tau_{\nu}-t_{\nu}\right) d t_{\nu},
\end{aligned}
$$

and the mean intensity is given by

$$
J_{\nu}\left(\tau_{\nu}\right)=\frac{1}{2} \int_{0}^{\infty} S_{\nu} E_{1}\left(\left|t_{\nu}-\tau_{\nu}\right|\right) d t_{\nu}
$$

where the exponential integral $\left(E_{n}(t)\right)$ is given by

$$
E_{n}(t)=\int_{1}^{\infty} \frac{e^{-t x}}{x^{n}} d x=\int_{0}^{1} e^{-t / \mu} \mu^{n-2} d \mu \quad(t>0) .
$$

\section{(c) Source Function}

Concerning LTE, the source function with thermal emission plus an isotropic coherent scattering component has the form

$$
S_{\nu}\left(\tau_{\nu}\right)=\frac{k_{\nu}}{k_{\nu}+\sigma_{\nu}} B_{\nu}\left(\tau_{\nu}\right)+\frac{\sigma_{\nu}}{k_{\nu}+\sigma_{\nu}} J_{\nu}\left(\tau_{\nu}\right),
$$

and if we introduce,

$$
\epsilon_{\nu}\left(\tau_{\nu}\right)=\frac{\sigma_{\nu}}{k_{\nu}+\sigma_{\nu}}
$$

then the source function becomes

$$
S_{\nu}\left(\tau_{\nu}\right)=\left(1-\epsilon_{\nu}\left(\tau_{\nu}\right)\right) B_{\nu}\left(\tau_{\nu}\right)+\epsilon_{\nu}\left(\tau_{\nu}\right) J_{\nu}\left(\tau_{\nu}\right),
$$

where $B_{\nu}\left(\tau_{\nu}\right)$ is the monochromatic Planck function.

\section{(d) Radiative Equilibrium and Temperature Correction}

Following the procedures outlined in the previous sections, the physical variables $\left(P_{g}, P_{e}, k_{\nu}, \sigma_{\nu}\right)$ and the radiation field $\left(F_{\nu}, J_{\nu}\right)$ have been calculated. The next step is to examine whether the model satisfies the condition of the radiative equilibrium or not. The radiative equilibrium condition in the form

$$
F(\tau)=\int_{0}^{\infty} F_{\nu} d \nu=\frac{\sigma T_{e f f}^{4}}{\pi}=\text { constant }
$$

implies that the integrated flux $F(\tau)$ remains constant as $\tau \Longrightarrow 0$. To achieve this condition we must improve the temperature structure of the model. For this purpose we adopt Simonneau and Crivellari (1988) procedure which could be outlined in the following paragraphs.

An alternative form of the condition of radiative equilibrium can be written as

$$
4 \pi \int_{0}^{\infty} \chi_{\nu}\left[J_{\nu}(\tau)-S_{\nu}(\tau)\right] d \nu=-Q(\tau)
$$

where $\chi_{\nu}=k_{\nu}+\sigma_{\nu}$ and $Q(\tau)=0$ if the condition is fulfilled exactly, in this method $Q(\tau)$ express the departure from radiative equilibrium.

The new temperature follows from the equation

$$
T^{4}(\tau)=\frac{\pi \alpha(\tau) J(\tau)}{\sigma_{R}}+\frac{Q(\tau)}{4 \sigma_{R} a_{P}(\tau)},
$$

where,

$$
\alpha(\tau) \equiv a_{J}(\tau) / a_{P}(\tau)
$$

$$
a_{P}(\tau) \equiv \int_{0}^{\infty} k_{\nu}(\tau) B_{\nu}(\tau) d \nu / \int_{0}^{\infty} B_{\nu}(\tau) d \nu
$$




$$
a_{J}(\tau) \equiv \int_{0}^{\infty} k_{\nu}(\tau) J_{\nu}(\tau) d \nu / \int_{0}^{\infty} J_{\nu}(\tau) d \nu
$$

$J(\tau)$ is the integrated mean intensity and $\sigma_{R}$ is the Stefan-Boltzman constant.

\section{COMPUTATIONAL METHODS}

\section{(a) Model Calculation \\ i) Initial Values}

To start the integration of the model we need the initial value of the gas pressure at the surface, so that we take $T=T_{g o}$ ( $T_{g o}$ is the grey boundary temperature ) and the initial value of the electron pressure is adopted as (Mihalas, 1967)

$$
\left(\log P_{e}\right)_{0}=-2.9+0.85 \log g-1.2 \theta_{0},
$$

where $\theta_{0}=\frac{5040}{T_{g 0}}$. Consequently $\log P_{g}$ is computed using the formula (Mihalas, 1978),

$$
\log P_{g}=\log P_{e}+\log \left[1+\frac{\sum \alpha_{i}}{\sum_{i} \alpha_{i} \sum_{j} j f_{i j}}\right]
$$

where, $\mathrm{j}$ is the stage of ionization, $\alpha_{i}$ is the abundance of the element $\mathrm{i}$ and $f_{i j}$ is given by

$$
f_{i j}=\frac{\prod_{k=0}^{j} \phi_{k}}{\sum_{k=0}^{i} \prod_{i=0}^{k} \phi_{i} r}
$$

where $\phi$ is known as Saha equation.

The initial value of the optical depth is computed using the hydrostatic equation in the form

$$
\frac{d \tau}{d \log P_{g}}=\frac{2.302585 P_{g}}{\frac{g}{\left(k_{\nu}+\sigma_{\nu}\right)_{s}}-\frac{d P_{r}}{d \tau}},
$$

which could be solved using Rung-Kutta method . Following the procedure mentioned above, $k_{\nu}\left(\theta_{0}, P_{e}\right)$, $\sigma\left(\theta_{0}, P_{e}\right)$ and $P_{g}\left(\theta_{0}, P_{e}\right)\left(\theta_{0}=5040 / T_{g 0}\right)$ at the starting optical depth will be determined.

\section{ii) Physical Variables}

After having the initial values required for integrating the model, the integration have been continued using Equation (1) with the approximate non-grey temperature distribution adopted by Hasegawa and Uesugi (1967) given by

$$
\begin{gathered}
T^{4}(\tau)=\frac{3}{4} T_{e f f}^{4}\left(1-\frac{1}{c}\right) \log \left(2 e^{\tau}-1\right)+T_{g}^{4}(\tau), \\
T_{g}^{4}(\tau)=\frac{3}{4} T_{e f f}^{4}[\tau+q(\infty)]
\end{gathered}
$$

$c=\alpha_{g} / \alpha, \alpha_{g}=\sqrt{3} / 4, \alpha=T_{g}^{4} / T_{\text {eff }}, q(\infty)=0.710446$.

Having the run of the physical variables as a function of the optical depth, we can compute tables of $k_{\nu}$ and $\frac{\sigma_{\nu}}{k_{\nu}+\sigma_{\nu}}$ at each depth for each frequency to be considered in the solution of the transfer equation. Now we can establish the optical depth scale at each frequency from the expression

$$
\tau_{\nu}(\tau)=\int_{0}^{\tau} \frac{k_{\nu}(t)+\sigma_{\nu}(t)}{\left[k_{\nu}(t)+\sigma_{\nu}(t)\right]_{s}} d t
$$

which is carried out by numerical integration.

\section{(b) Solution of the Transfer Equation}

As a result of having the opacities and optical depths at each frequency, we are in the situation which enables us to solve the equation of transfer. In our code we follow the method adopted by Avrett and Loeser ( 1963). The source function is approximated by an interpolation formula that is valid between discrete points . Then the integral equation can be reduced to a set of linear algebraic equations, which may be solved by standard numerical methods.

If the source function can be represented by a series of equations

$$
S\left(\tau_{j}\right)=\sum_{i}^{N} a_{i} f_{i}\left(\tau_{j}\right),
$$

then inserting Equation (8) into Equation (23) and arranging the terms we get,

$$
\begin{array}{r}
\sum_{i}^{N} a_{i}\left[f_{i}\left(\tau_{j}\right)-\frac{\epsilon_{j}}{2} \int_{0}^{\infty} f_{i}(t) E_{1}\left|\tau_{j}-t\right| d t\right] \\
=\left(1-\epsilon_{j}\right) B\left(\tau_{j}\right),
\end{array}
$$

which may be solved for the $a_{i}$ 's. The quantities $S_{\nu}, J_{\nu}, F_{\nu}$ could be computed from the expressions,

$$
\begin{aligned}
S_{\nu}\left(\tau_{j}\right) & =\sum_{i}^{N} a_{i} G F_{i}\left(\tau_{j}\right), \\
J_{\nu}\left(\tau_{j}\right) & =\sum_{i}^{N} a_{i} G J_{i}\left(\tau_{j}\right), \\
F_{\nu}\left(\tau_{j}\right) & =\sum_{i}^{N} a_{i} G H_{i}\left(\tau_{j}\right) .
\end{aligned}
$$

Following Avrett and Loeser (1963) the function $G F_{i}$ is given by

$$
\begin{aligned}
& G F_{1}(x)=1, \\
& G F_{i}(x)=\left[\begin{array}{c}
\left(1-\frac{x}{x_{i}}\right)\left(1-\frac{\lambda x}{x_{i}}\right), 0 \leq x \leq x_{i} \\
0, x>x_{i}, i=2,3, \ldots(n-1)
\end{array}\right], \\
& G F_{n}(x)=x,
\end{aligned}
$$


where $0 \geq \lambda \leq 1$.

With this choice of $G F_{i}$, the quantities $G J_{i}$ and $G H_{i}$ have the form

$$
G J_{i}\left(\tau_{j}\right)=\frac{1}{2} \int_{0}^{\infty} f_{i}(t) E_{1}\left(\left|\tau_{j}-t\right|\right) d t
$$

and

$$
\begin{aligned}
G H_{i}\left(\tau_{j}\right) & =\frac{1}{2} \int_{\tau_{j}}^{\infty} f_{i}(t) E_{2}\left(t-\tau_{j}\right) d t \\
& -\frac{1}{2} \int_{0}^{\tau_{j}} f_{i}(t) E_{2}\left(\tau_{j}-t\right) d t .
\end{aligned}
$$

We have examined several methods for computing $G J_{i}$ and $\mathrm{GH}_{i}$. From these comparisons using the grey source function

$$
S=3[\tau+q(\tau)]
$$

we found that the analytic expressions provided by Klinglesmith (1967), after some necessary corrections are the most accurate one (improved expression would be found in Appendix).

At this stage we have obtained the source function, mean intensity and flux integral at each frequency for each optical depth.

\section{(c) Computational Algorithm}

In what follows, a computational algorithm for model atmosphere calculation will be established.

- Input : $T_{e f f}, \log g$.

- Computational Sequence :

1. Compute the surface temperature using Equation (20).

2. Compute the starting optical depth using Equation (19).

3. Compute the starting physical parameters $\left(P_{g}\right)_{0},\left(P_{e}\right)_{0}$ using Equations (17) and (18).

4. Integrate Equation (1) to compute $P_{g}$ at the selected optical depth $\tau$.

5. Compute tables of $k_{\nu}$ and $\sigma_{\nu} /\left(k_{\nu}+\sigma_{\nu}\right)$.

6. Integrate Equation (22) to compute the monochromatic optical depth.

7. Compute the monochromatic flux and mean intensity from Equations (26).

8. Test the radiative equilibrium condition using Equation (11).

9. If the previous condition is satisfied the model converge, if not go to step 10.

10. Compute the new temperature structure using Equation (13) and go to step 4.

- The algorithm is completed.

\begin{tabular}{|c|c|c|}
\hline Models & $T_{e f f}(K)$ & $\log g$ \\
\hline Model I & 25000 & 6 \\
\hline Model II & 30000 & 6 \\
\hline Model III & 30000 & 4 \\
\hline
\end{tabular}

TABLE 1.

PARAmeters OF THE COMPUTED MODELS, ALL MODELS ARE PURE HELIUM.

TABLE 2

ThE RATIO $\frac{T_{0}}{T_{e f f}}$

\begin{tabular}{ccc}
\hline$T_{\text {eff }}$ & $\log g$ & $\frac{T_{0}}{T_{\text {eff }}}$ \\
\hline 25000 & 6 & 0.6547 \\
30000 & 6 & 0.6180 \\
30000 & 4 & 0.6399 \\
\hline
\end{tabular}

\section{RESULTS AND DISCUSSION}

Following the method of computation described above, three models were computed with the parameters listed in Table 1.

The calculation will concerned only for the pure helium atmosphere. We consider only bound-free and free-free transitions of HeI and HeII and Thompson electron scattering. Line blanketing, which is due mainly to the resonance line $\lambda 504 \AA$ of HeI is neglected as well as the Raleigh scattering.

\section{(a) Temperature Depth Relationship}

After altering the temperature distribution to obtain the flux constancy, the form of the temperature-depth relation now has some interest. First the ratio $T_{0} / T_{\text {eff }}$ (the surface temperature to the effective temperature ratio) has been altered due to the iteration (the ratio for the grey case is 0.811 ). The results obtained from the models are given in Table 2 .

We see from the table the pronounced deviation from the grey ratio. The condition of the radiative equilibrium implies that the integrated flux,

$$
\begin{gathered}
\int_{0}^{\infty} d \nu \int_{\tau_{\nu}}^{\infty} S_{\nu}\left(\tau_{\nu}\right) E_{2}\left|\tau_{\nu}-t\right| d \tau_{\nu} \\
-\int_{0}^{\infty} d \nu \int_{0}^{\tau_{\nu}} S_{\nu}\left(\tau_{\nu}\right) E_{2}\left|t-\tau_{\nu}\right| d \tau_{\nu}
\end{gathered}
$$

remains constant as $\tau \Rightarrow 0$. As $\tau$ increases by small amounts, it is evident from the above equation that radiative equilibrium in the outer parts of the atmosphere could be achieved with considerable drops in the temperature at $\tau \Rightarrow 0$. This is because the helium opacity in the outer layers is highly depending on frequency, where the main contribution to it is the true absorption i.e. the atmosphere in these regions is non-grey. 


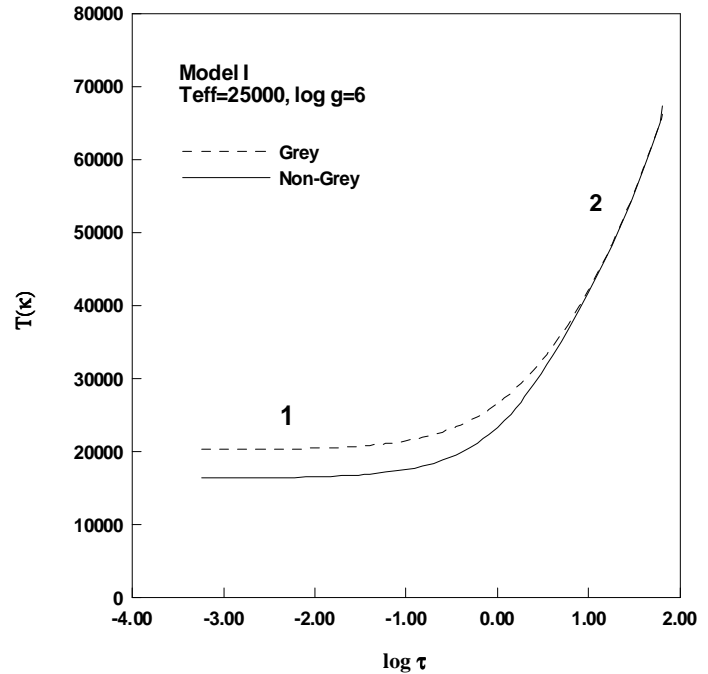

Fig. 1.- Temperature depth relation for Model I.

This could be clearly noticed from the results obtained and represented in Figures 1, 2 and 3, the part labelled 1 in the figures.

On the other hand when $\tau$ increases considerably, the first integral in Equation (30) becomes too large leading to an increase in the integrated flux $F(\tau)$, while the second integral is strongly affected causing marked decrease in $F(\tau)$. At these large optical depths, where the temperatures are very high, the main contribution to the opacity is the scattering, i.e. it is independent of frequency, then the atmosphere in this region is a grey one. The final result confines in a small change in temperature, the part labelled 2 in the figures.

\section{(b) UBV Colors}

To compute U-B, B-V and the bolometric correction for the energy distribution of the models we proceed as follows:

- By using the filter function $(S(\lambda))$ published by Matthews and Sandage (1963) and the emergent monochromatic flux $(F(\lambda))$, we compute $\mathrm{u}-\mathrm{b}, \mathrm{b}-\mathrm{v}$ from the expressions,

$$
u-b=-2.5 \log \frac{\int_{0}^{\infty} S_{b}(\lambda) F(\lambda) d \lambda}{\int_{0}^{\infty} S_{u}(\lambda) F(\lambda) d \lambda},
$$

and

$$
v-b=-2.5 \log \frac{\int_{0}^{\infty} S_{v}(\lambda) F(\lambda) d \lambda}{\int_{0}^{\infty} S_{b}(\lambda) F(\lambda) d \lambda},
$$

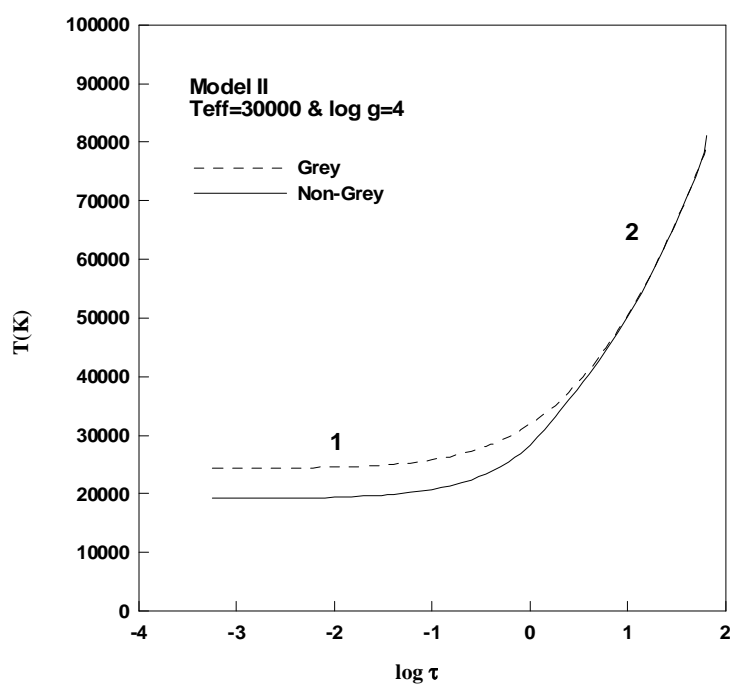

Fig. 2. - Temperature depth relation for Model II.

TABLE 3.

Colors And Bolometric CORRECTIONS OF THE MODELS

\begin{tabular}{ccccc}
\hline$T_{e f f}$ & $\log g$ & $\mathrm{U}-\mathrm{B}$ & $\mathrm{B}-\mathrm{V}$ & $\mathrm{B.C}$ \\
\hline 25000 & 6 & -1.0882 & -0.229 & -3.506 \\
30000 & 6 & -1.1237 & -0.269 & -3.369 \\
30000 & 4 & -1.1333 & -0.278 & -3.296 \\
\hline
\end{tabular}

- Compute (U-B) and (B-V) by using the following equations:

$$
\begin{gathered}
U-B=0.921(u-b)-1.308 \\
B-V=1.024(b-v)+0.81
\end{gathered}
$$

- The bolometric correction could be given by

$$
B . C=2.5 \log \frac{\int_{0}^{\infty} F(\lambda) S_{V}(\lambda) d \lambda}{\int_{0}^{\infty} F(\lambda) d \lambda}+C_{1},
$$

where $C_{1}=0.95 \pm 0.01 \mathrm{mag}$ (Code et al, 1976).

The $(U-B),(B-V)$, and $B . C$ for each model is tabulated in Table 3.As we see from the table the dependence on the temperature is clear but the dependence on the gravity is slight.

The model with $T_{\text {eff }}=30000$ and $\log g=4$ is compared with the main sequence model of Mihalas and the model of pure helium produced by Klinglismith (1967) at the same parameters. The comparison is shown in Table 4.and reveals a good results. 


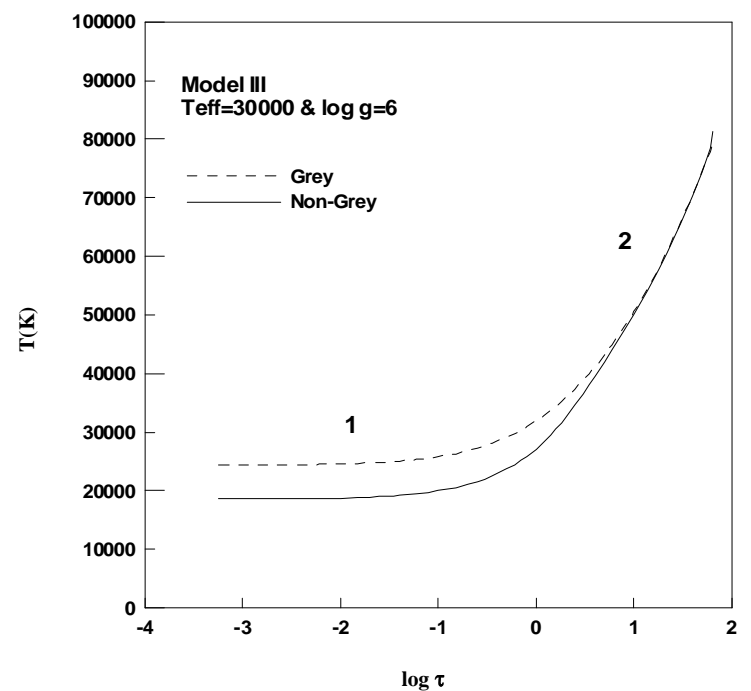

Fig. 3.- Temperature depth relation for Model III.

TABLE 4.

COMPARISON OF COLORS FOR MODEL III

\begin{tabular}{ccc}
\hline Model & U-B & B-V \\
\hline Mihalas & -1.15 & -0.29 \\
Klinglismith & -1.11 & -0.29 \\
Model III & -1.13 & -0.28
\end{tabular}

\section{CONCLUSION}

As a summarization of the paper, we elaborated an independent FORTRAN code for calculating LTEplane-parallel model atmospheres.

The transfer equation has been solved using Avrett and Loeser method. It is shown that, using an approximate non-gray temperature distribution together with the iteration factors method (Simonneau and Crivellari, 1988) for correcting the temperature distribution reduce the number of iteration required to achieve the condition of radiative equilibrium. The computational procedure used is discussed in a stepwise fashion to clarify the code.

Preliminary results for pure helium model atmospheres are presented. Three models have been computed to test the physical properties of the atmospheres. Application to the line blanketing model atmospheres will be followed in a series of papers.

\section{REFERENCES}

Auer L. H., Mihalas D., 1972, Non-Lte Model Atmospheres. VII. The Hydrogen and Helium Spectra of the O Stars, ApJS 24, 193
Avrett, E. H. \& Loeser, R., 1963, A simple and accurate method for the evaluation of the Milne integrals, JQSRT, 3, 201

Code, A. D, Davis, J, Bless, R. C, \& Brown, R. H., 1976, Empirical effective temperatures and bolometric corrections for early-type stars, ApJ, 203, 417

Hasegawa, Y. \& Uesugi, A., 1967, Memoirs of the College of Science, Unversity of Kyoto, Series A, vol.31, 303-309.

Hubeny I., 1997, in Stellar Atmospheres: Theory and Observations, eds. De Gréve J. P., Blomme R., Hensberge H., Springer, p. 1.

Klinglesmith, D. A., 1967, Ph.D, Indiana University.

Kudritzki R. P., 1997, Quantitative Spectroscopy of the Brightest Blue Supergiant References 23 Stars in Galaxies in Proceedings of the 8th Canary Winter School: Stellar Astrophysics for the Local Group, a First step to the Universe. eds. Aparicio A., Herrero A., Cambridge University Press.

Kurucz, R., 1979, Model atmospheres for G, F, A, B, and O stars, ApJS, 40, 1.

Matthews, T. A. \& Sandage, A. R., 1963, Optical Identification of 3c 48, 3c 196, and 3c 286 with Stellar Objects, ApJ, 138, 30

Mihalas, D., 1967, Methods In Computational Physics, Volume 7, Astrophysics, Academic Press.

Mihalas, D., 1978, Stellar Atmospheres, W. H Freeman and Company.

Simonneau, E. \& Crivellari, L., 1988, On the application of iteration factors for temperature correction in stellar atmospheres, ApJ, 330, 415

Underhill, A., 1949, On the Effect of Radiation Pressure in the Atmospheres of Early-Type Stars, MNRAS, 109, 562 


\section{Appendix: An Improved Analytic Expressions for the $G J$ and $G H$ Matrices}

The analytic expressions suggested by Klinglesmith (1967) will be treated in the following manner. From the nature of the Avrett parabolas there are four cases that need to be considered in computing the $G J$ and $G H$ matrices. The general expression can be written as

$$
\begin{array}{r}
G_{k}\left(i, \tau_{j}\right)=\frac{1}{2} \int_{\tau_{j}}^{\infty} f_{i}(t) E_{k}\left(t-\tau_{j}\right) d t \\
+(-1)^{k+1} \frac{1}{2} \int_{0}^{\tau_{j}} f_{i}(t) E_{k}\left(\tau_{j}-t\right) d t
\end{array}
$$

where $k=1$ implies $G J$ and $k=2$ implies $G H$

The four cases are:

I: $i=1$ and all $j$

II: $i=N$ and all $j$

III: $j<i$

IV $: j \geq i$

The resultant four expressions are:

Case I:

$$
\begin{array}{r}
G_{k}\left(1, \tau_{j}\right)=\frac{1}{2} \int_{\tau_{j}}^{\infty} E_{k}\left(t-\tau_{j}\right) d t \\
+(-1)^{k+1} \frac{1}{2} \int_{0}^{\tau_{j}} E_{k}\left(\tau_{j}-t\right) d t
\end{array}
$$

Case II:

$$
\begin{array}{r}
G_{k}\left(N, \tau_{j}\right)=\frac{1}{2} \int_{\tau_{j}}^{\infty} t E_{k}\left(t-\tau_{j}\right) d t \\
+(-1)^{k+1} \frac{1}{2} \int_{0}^{\tau_{j}} t E_{k}\left(\tau_{j}-t\right) d t
\end{array}
$$

Case III:

$$
\begin{gathered}
G_{k}\left(i, \tau_{j}\right)=\frac{1}{2} \int_{\infty}^{\tau_{j}} H E_{k}\left(t-\tau_{j}\right) d t \\
+(-1)^{k+1} \frac{1}{2} \int_{0}^{\tau_{j}} H E_{k}\left(\tau_{j}-t\right) d t,
\end{gathered}
$$

where

$$
H=\left(1-\frac{t}{t_{i}}\right)\left(1-\frac{\lambda t}{\tau_{j}}\right) .
$$

Case IV:

$$
G_{k}\left(i, \tau_{j}\right)=(-1)^{k+1} \frac{1}{2} \int_{0}^{\tau_{j}} H E_{k}\left(\tau_{j}-t\right) d t
$$

The limits on Case III and Case IV are a result of the fact that for $j \geq 1, f_{i}\left(\tau_{j}\right)=0$. By making a transformation of variables so that the argument of the exponential integral is the integration variable, these expressions can be integrated easily. The transformation for $t-\tau_{j}$ is

$$
\begin{array}{r}
x=t-\tau_{j}, \\
d x=d t, \\
t=x+\tau_{j} .
\end{array}
$$

The transformation for $\tau_{j}-t$ is

$$
\begin{gathered}
y=\tau_{j}-t, \\
d y=-d t \\
t=\tau_{j}-y
\end{gathered}
$$

Since at worst $f_{i}\left(\tau_{j}\right)$ is a parabola there are three types of integrals that need to be considered:

$$
\begin{aligned}
& \int E_{k}(x) d x=-E_{k+1}(x) \\
& \int x E_{k}(x) d x=-\left[x E_{k+1}(x)+E_{k+2}(x)\right] \\
& \int x^{2} E_{k}(x) d x=-\left[x^{2} E_{k+1}(x)+2 x E_{k+2}(x)+2 E_{k+3}\right]
\end{aligned}
$$

After making the transformations of variables and arranging the terms in powers of the integration variable, the four cases become Case I:

$$
\begin{array}{r}
G_{k}\left(1, \tau_{j}\right)=\frac{1}{2} \int_{\tau_{j}}^{\infty} E_{k}\left(t-\tau_{j}\right) d t \\
+(-1)^{k+1} \frac{1}{2} \int_{0}^{\tau_{j}} E_{k}\left(\tau_{j}-t\right) d t
\end{array}
$$

Case II:

$$
\begin{array}{r}
G_{k}\left(1, \tau_{j}\right)=\frac{1}{2} \int_{\tau_{j}}^{\infty}\left(\tau_{j}+x\right) E_{k}(x) d x \\
+(-1)^{k+1} \frac{1}{2} \int_{0}^{\tau_{j}}\left(\tau_{j}-y\right) E_{k}(y) d y
\end{array}
$$

Case III:

$$
\begin{aligned}
G_{k}\left(i, \tau_{j}\right)= & \frac{1}{2} \int_{0}^{\left(\tau_{i}-\tau_{j}\right)} Q 1 E_{k}(x) d x+ \\
& (-1)^{k+1} \frac{1}{2} \int_{0}^{\tau_{j}} Q 2 E_{k}(y) d y
\end{aligned}
$$

where

$Q 1=1-\frac{1+\lambda}{\tau_{i}} \tau_{j}+\frac{\lambda \tau_{j}^{2}}{\tau_{i}^{2}}+\left[\frac{\lambda-1}{\tau_{i}}+\frac{2 \lambda \tau_{j}}{\tau_{i}}\right] x+\frac{\lambda}{\tau_{i}^{2}} x^{2}$,

and

$Q 2=1-\frac{1+\lambda}{\tau_{i}} \tau_{j}+\frac{\lambda \tau_{j}^{2}}{\tau_{i}^{2}}+\left[\frac{1+\lambda}{\tau_{i}}-\frac{2 \lambda \tau_{j}}{\tau_{i}}\right] y+\frac{\lambda}{\tau_{i}^{2}} y^{2}$.

Case IV:

$$
G_{k}\left(i, \tau_{j}\right)=(-1)^{k+1} \frac{1}{2} \int_{\tau_{j}}^{\left(\tau_{j}-\tau_{i}\right)} Q 2 E_{k}(y) d y
$$


The results of these integrations for $\lambda=1$ give: Case I:

$$
\begin{gathered}
G_{1}\left(1, \tau_{j}\right)=G J_{1 J}=1-\frac{1}{2} E_{2}\left(\tau_{j}\right) \\
G_{2}\left(1, \tau_{j}\right)=G H_{1 J}=\frac{1}{2} E_{3}\left(\tau_{j}\right)
\end{gathered}
$$

Case II: $i=1$ for all $j$

$$
\begin{aligned}
& G_{1}\left(N, \tau_{j}\right)=G J_{N, J}=\tau_{j}+\frac{1}{2} E_{3}\left(\tau_{j}\right) \\
& G_{2}\left(N, \tau_{j}\right)=G H_{N, J}=\frac{1}{3}-\frac{1}{2} E_{4}\left(\tau_{j}\right)
\end{aligned}
$$

Case III: $j<i$

$$
\begin{aligned}
G_{1}\left(i, \tau_{j}\right)=G J_{i, j}= & Q 3 \frac{1}{\tau_{i}} E_{3}\left(\tau_{j}\right)-\frac{1}{\tau_{i}^{2}} E_{4}\left(\tau_{i}-\tau_{j}\right) \\
& +E_{4}\left(\tau_{j}\right)+\frac{1}{2} E_{3}\left(\tau_{i}\right)-\frac{1}{2} E_{2}\left(\tau_{j}\right)
\end{aligned}
$$

where

$$
Q 3=1-\frac{2 \tau_{j}}{\tau_{i}}+\frac{1}{\tau_{i}^{2}}\left[\tau^{2}+\frac{2}{3}\right]
$$

and

$$
\begin{array}{r}
G_{2}\left(i, \tau_{j}\right)=G H_{i, j}=\frac{1}{2} E_{3}\left(\tau_{j}\right)+\frac{1}{\tau_{i}} E_{4}\left(\tau_{j}\right)+ \\
\frac{1}{\tau_{i}} E_{5}\left(\tau_{j}\right)\left[-E_{5}\left(\tau_{j}-\tau_{i}\right)+\frac{2 \tau_{j}}{3 \tau_{i}}\right]-\frac{2}{3 \tau_{i}}
\end{array}
$$

Case IV $: j \geq i$

$$
\begin{aligned}
& G_{1}\left(i, \tau_{j}\right)=G J_{i, j}=\frac{1}{2} E_{2}\left(\tau_{j}\right)+\frac{1}{\tau} E_{3}\left(\tau_{j}\right)- \\
& \frac{1}{2}\left[E_{4}\left(\tau_{j}-\tau_{i}\right)-E_{4}\left(\tau_{j}\right)\right] \\
& G_{2}\left(i, \tau_{j}\right)=G H_{i, j}=-\frac{1}{2} E_{3}\left(\tau_{j}\right)-\frac{1}{\tau} E_{4}\left(\tau_{j}\right)- \\
& \frac{1}{\tau_{i}^{2}}\left[E_{5}\left(\tau_{j}-\tau_{i}\right)-E_{5}\left(\tau_{j}\right)\right]
\end{aligned}
$$

